\title{
Exposure and response to methyl isocyanate: results of a community based survey in Bhopal
}

\author{
N ANDERSSON,' M KERR MUIR, ${ }^{2}$ V MEHRA, ${ }^{3}$ A G SALMON' \\ From the Department of Occupational Health,' London School of Hygiene and Tropical Medicine, London \\ WCIE 7HT, Moorfields Eye Hospital, ${ }^{2}$ London ECI 2PD, UK, and Chattisgarh Eye Hospital, ${ }^{3}$ Raipur, MP, \\ India
}

ABSTRACT In the two weeks immediately after the Bhopal disaster a community based survey was carried out in a series of eight exposed and two non-exposed clusters of households. The primary concern was the effect of the gas (subsequently identified as methyl isocyanate) on the eyes of the victims but data were also sought on respiratory status and the first symptoms of the exposure. No case of blindness was encountered that could be attributed to the gas. The most frequent symptoms reported were burning of the eyes, coughing, watering of the eyes, and vomiting. Among these, the frequency of cough most closely followed the rate of death in the different clusters. Although much rarer overall, the frequency of reported diarrhoea appeared to bear a stronger relation to death rates. Reports of photophobia and the clinical finding of superficial interpalpebral erosion of the cornea were more frequent where the death rates were lower. This clinical and epidemiological picture is consistent with different effects of the gas at different doses (as estimated from distance from the factory).

The release of a gas cloud from the Union Carbide factory at Bhopal, India, on the night of $2 / 3$ December 1984 resulted in numerous deaths and injuries among the surrounding population. It is now believed that the immediate cause of the disaster was that water entered a tank where 41 tonnes of methyl isocyanate (MIC) were stored, causing a runaway chemical reaction. The heat of the reaction, possibly augmented by reactions with other materials present in the tank as contaminants, produced vapourisation of such momentum that it could not be contained by the safety systems, which may in any event have been defective or of inadequate capacity (S Varadarajah, report to the Council of Scientific and Industrial Research, India, 1985). There is still considerable confusion about what effects among the survivors can be attributed to exposure to the gas. In the two weeks immediately after the disaster a team of Indian and British doctors carried out a formal community based epidemiological study of exposed and unexposed populations before the mass exodus from the city.

\section{Methods}

Several of the worst cases in two hospitals were examined in the days immediately after the disaster to obtain an initial indication of the nature and severity

Accepted 22 June 1987 of effects among survivors. Results of slit lamp examination by two ophthalmologists were reported earlier. ${ }^{2}$ A preliminary assessment of the area of exposure and the effects suffered was made by talking to people who lived at varying distances and directions from the plant. From this it was possible to map out preliminary exposure zones according to reports of human and animal deaths, symptoms, and perceptions of the presence of the gas. Cases seen in the community before the start of formal survey were not included in the study, although they provided valuable information regarding the limits and nature of the exposure.

Eight housing clusters were selected with the exposure zone, covering a range of different severities of exposure as judged by the preliminary inquiries. A household survey was carried out in the second week after the release of the gas. Two non-exposed clusters were selected, having apparently similar socioeconomic status to the exposed groups but located 14 and $17 \mathrm{~km}$ away from the plant, where no one reported symptoms of gas exposure within several kilometers. Starting with one arbitrary selection, houses were contacted consecutively in each cluster, moving from door to door with a questionnaire drawn up for the purpose. All inhabitants present in each dwelling at the time of the visit were examined following a standard format. The British doctors used an interpreter. The initial survey lasted from 5 to 12 December, after which time the entire exposure area was 
deserted as people left in case the plant was restarted to "neutralise" the remaining stock of MIC.

The first survey day was spent by the participating doctors practising the administration of the questionnaire and the examination in an attempt to ensure consistency. The questionnaire inquired about name, age, duration of exposure based on experience of acute symptoms and presence in the exposed areas, deaths in the household, the first four symptoms perceived after the exposure, eye symptoms, and treatment received to date. The first four symptoms were requested without prompting by the interviewer, whereas for the eye symptoms a checklist was read out after the first four symptoms had been recorded. A rapid clinical assessment of respiratory signs was recorded (obvious tachypnoea, audible wheeze, use of accessory muscles) so far as possible without alerting the interviewee to this. The initial focus of the inquiry was the eye consequences and to lay a baseline for future possible deterioration of sight.

Data were coded and sorted using the standard SPSS statistical package. Analysis was done using the Mantel Haenszel procedure for combining data from different strata ${ }^{3}$ and the Mantel extension of this test. ${ }^{4}$ Statistical results are reported as the chi-squared statistic, ${ }^{2}$ odds ratio (OR), and confidence interval (CI) for a stated level of probability

\section{Results}

Examination of severely affected hospital inpatients indicated that the main symptoms which were considered life threatening were those involving the respiratory system. The worst eye cases brought forward for slit lamp examination at Ghandi Medical College by two ophthalmologists showed no case of deep corneal damage. ${ }^{2}$

Travel by foot, tricab, and taxi allowed the outer limits of the exposure zones to be mapped out, as shown in the figure. People were affected (mostly burning eyes and coughing) over $1 \mathrm{~km}$ north of the factory, despite the light north wind. No deaths were reported to the north of the factory. People from this area tended to flee northward, rapidly getting away from the gas cloud. For analytical purposes, data were available from questionnaires of 379 people in the eight exposed clusters and 119 people in the two unexposed clusters (an average $2.32 \%$ of the estimated population in the area surrounding the study clusters). All study clusters were south of the factory. Table 1 shows the age distributions in these clusters. The age distributions of exposed and unexposed groups show some differences, which are most pronounced in the extremes of age. A reporting bias was noted in exposure cluster 7 , a factory compound, where men conspicuously understated their ages relative to the estimate of the interviewers (presumably to protect their employment). Sex ratio in the two populations was similar (exposed group $41.7 \%$ women, unexposed group $42.9 \%$ women). The sex of about $15 \%$ of respondents in each group was not recorded.

The duration of exposure across all the exposed groups $(n=379)$ was as follows: $1 \%$ left the areas in which symptoms of gas exposure were reported within one hour, $15 \%$ were exposed for one hour, $16 \%$ between one and two hours, $16 \%$ for two hours, $8 \%$ for two to three hours, and $2 \%$ for three hours. A further $29 \%$ said that they had been present in the area throughout the exposure period (estimated to be about four hours). No details regarding individual exposure were obtained on $13 \%$, three quarters of whom were children under the age of 10 years. Between clusters where deaths occurred (1 to 6) and the remaining two exposure clusters there was no apparent difference in the average duration of exposure nor in the proportion who fled from the exposed area $\left(\chi^{2}=0 \cdot 25\right)$.

Eack. interviewee was asked how many people had died in their household. The results of this are shown in table 2. Deaths were recorded in clusters 1, 2, 3, 5, and 6. Numerous deaths were known to have occurred in cluster 4 , in households where there were no survivors, but the limitations of the study method meant that these numbers could not be recorded on a questionnaire. A total of 51 deaths was reported in the houses where the 379 survivors were examined. Based on the estimated population in and around each cluster (800 in 6: 1500 in 1: 2000 each in 2, 3, and 7: 3000 each in 4 and 5: and 4000 in 8 ), it is possible to calculate that about 1850 deaths occurred in and around the eight clusters used in this study. This covered about $70 \%$ of the exposed area.

The most frequently mentioned first symptom among the survivors in all the study clusters was burning eyes. In answer to the open question regarding sequence of symptoms, $56 \%$ mentioned burning eyes first, $15 \%$ mentioned it second, $7 \%$ third, and $3 \%$ as the fourth symptom (total $81 \%$ ). The second most commonly reported symptom was coughing. The next two most frequently mentioned symptoms were watering of the eyes and vomiting (see table 3 ).

Table 3 shows the relation between initial symptoms and death. It would appear that burning eyes do not have a particularly high reported prevalence in those clusters where death was common. The frequency of reporting cough as one of the first four symptoms, however, follows closely the distribution of deaths. This trend was significant at the $5 \%$ level $\left(\chi^{2}=4.32\right.$ using the Mantel extension as a test of trend with $1 \mathrm{DF}$ ). Using 25 mortality as a cut off point, clusters 1 , 2 , and 6 were strongly associated with reported cough $\left(\chi^{2}=8.39 ;\right.$ OR $\left.=2.17 ; 99.5 \% \mathrm{CI}=1.02-4.59\right)$. Diarrhoea and shortness of breath among the 


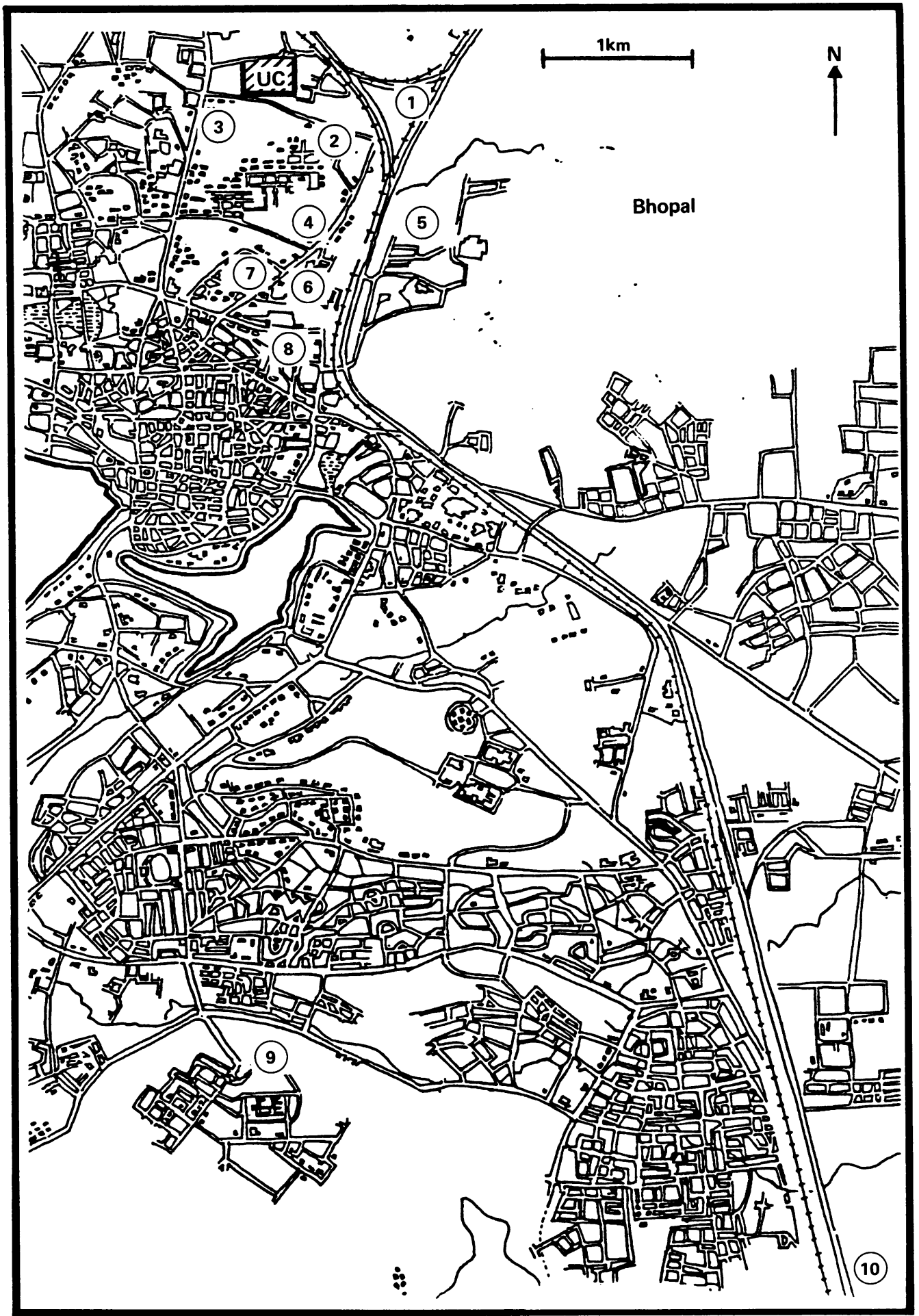

Figure Map of Bhopal showing location of Union Carbide plant (UC) and of exposed (1-8) and unexposed $(9,10)$ clusters. Scale is indicated by $1 \mathrm{~km}$ bar: area shown is about $6 \mathrm{~km} \times 9 \mathrm{~km}$. 
Table 1 Age distritbution in the study clusters

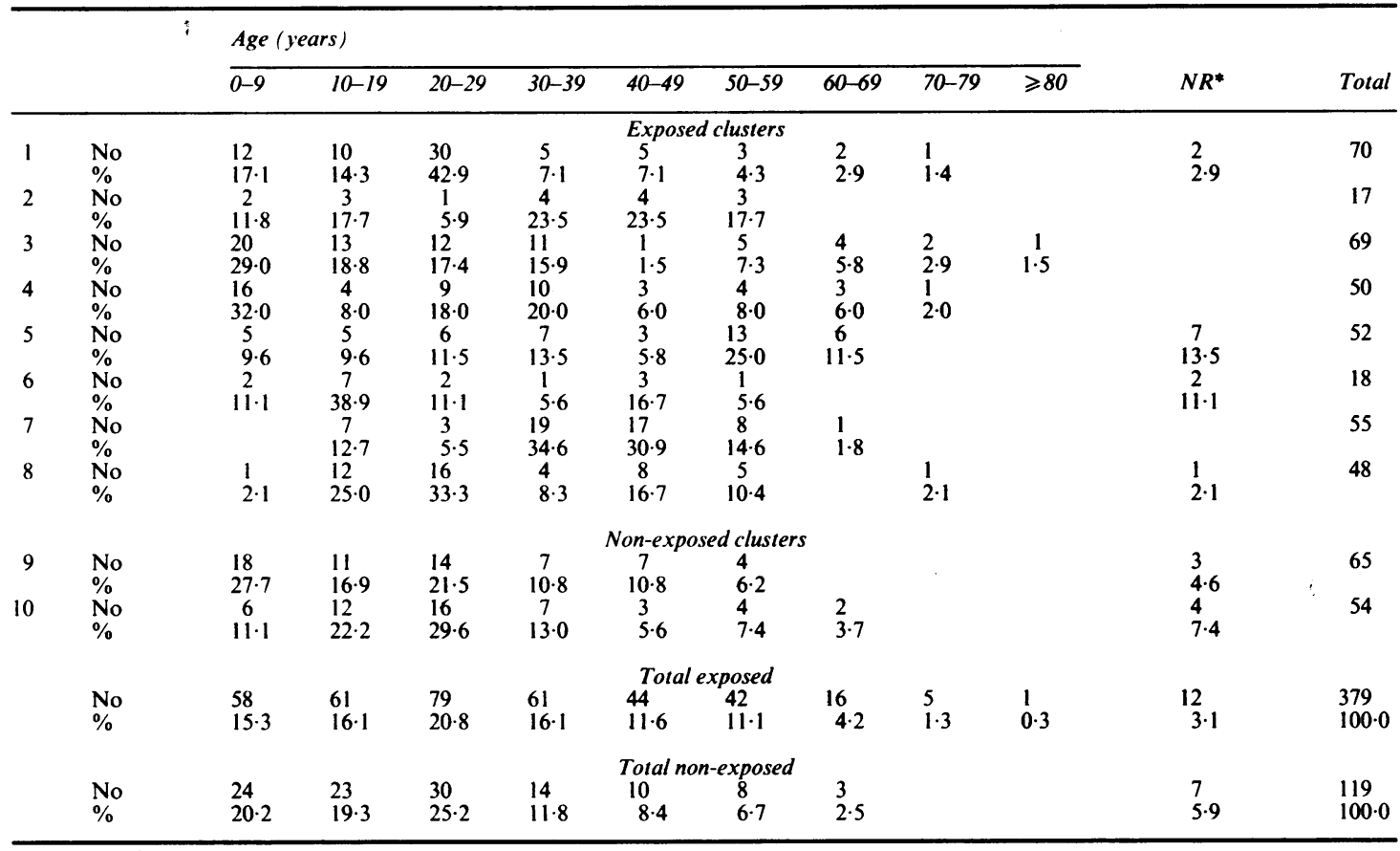

*NR, no reply.

survivors had a notable relation to the occurrence of death. Diarrhoea was reported with increasing frequency across clusters with increasing mortality $\left(\chi^{2}\right.$ $=10.3$ using the Mantel extension as a test of trend with 1 DF). Shortness of breath, on the other hand, showed a strongly inverse relation with clustering of deaths $\left(\chi^{2}=12 \cdot 3\right.$ by the same test).

Examination of the eyes showed different patterns in the various clusters (table 4). Red eyes were more common among the exposed compared with unexposed people overall, a finding that was statistically significant at the $90 \%$ confidence level $\left(\chi^{2}=3 \cdot 1\right.$; OR $=1.71 ; 90 \% \mathrm{CI}=1.04-2.08)$. The characteristic superficial interpalpebral erosion of the cornea and conjunctiva (SIPE) associated with exposure to MIC was observed in seven of the eight exposure groups with differing frequency. This sign was most frequent in clusters 4 and $6(36 \%$ and $50 \%$ respectively). These were the two clusters where the lowest proportion of people actually reported burning of the eyes as a symptom immediately after exposure. The possibility of the coincidence of SIPE with low reporting of burning eyes having occurred by chance may be excluded with greater than $99.9 \%$ confidence $\left(\chi^{2}=\right.$ 59.66 with $1 \mathrm{DF})$. The apparently inverse relation between burning and SIPE was not found between SIPE and reported watering of the eyes but it was apparent with photophobia and the sensation that the eyes could not be opened (see table 5). Survivors in the clusters where deaths had occurred (1-6) were nearly 12 times more at risk of SIPE than other exposed people: in clusters 1-6 there were 52 cases out of 224 survivors compared with two among 101 survivors in clusters 7 and $8\left(\chi^{2}=17 \cdot 49 ; \mathrm{OR}=11 \cdot 7 ; 99 \cdot 9 \% \mathrm{CI}=\right.$ $1 \cdot 69-81 \cdot 4)$.

There was no evidence of eye problems other than SIPE and redness in the exposure clusters compared with the non-exposure clusters $(9$ and 10$)$. There was no difference in the occurrence of corneal opacity $\left(\chi^{2}\right.$ $=0.05)$, Bitot spot $\left(\chi^{2}=0 \cdot 14\right)$, active infection $\left(\chi^{2}=\right.$ $0.83)$, and evidence of past trachoma $\left(\chi^{2}=0.13\right)$. Pterygium $\left(\chi^{2}=17.6 ;\right.$ OR $\left.=3 \cdot 3\right)$ and cataract $\left(\chi^{2}=\right.$

Table 2 Deaths by cluster

\begin{tabular}{llll}
\hline Cluster & $\begin{array}{l}\text { People } \\
\text { examined }\end{array}$ & $\begin{array}{l}\text { Households } \\
\text { with deaths }\end{array}$ & $\begin{array}{l}\text { No of } \\
\text { deaths }\end{array}$ \\
\hline 1 & 70 & 6 & 23 \\
2 & 17 & 3 & 10 \\
3 & 69 & 1 & 2 \\
4 & 50 & 2 & $-7^{\dagger}$ \\
5 & 52 & 1 & 7 \\
6 & 18 & 0 & 7 \\
7 & $55^{*}$ & 0 & 0 \\
8 & 48 & & 0
\end{tabular}

*Works compound: only men of working age examined.

IIn cluster 4 at least three households were encountered where deaths had occurred. These were not included in the survey. 
Table 3 Death rates in relation to early symptoms

\begin{tabular}{|c|c|c|c|c|c|c|c|c|}
\hline $\begin{array}{l}\text { Cluster } \\
\text { No }\end{array}$ & $\begin{array}{r}2 \\
17\end{array}$ & $\begin{array}{r}6 \\
18\end{array}$ & $\begin{array}{r}1 \\
70\end{array}$ & $\begin{array}{r}5 \\
52\end{array}$ & $\begin{array}{r}3 \\
69\end{array}$ & $\begin{array}{r}4 \\
50\end{array}$ & $\begin{array}{r}7 \\
55\end{array}$ & $\begin{array}{r}8 \\
48\end{array}$ \\
\hline Death rate* $(\%)$ & 37 & 28 & 25 & 15 & 3 & $t$ & 0 & 0 \\
\hline \multicolumn{9}{|l|}{ First four symptoms (\%): } \\
\hline Burning eyes & 76 & 60 & 86 & 83 & 74 & 60 & 95 & 94 \\
\hline Coughing & 88 & 78 & 77 & 58 & 65 & 64 & 65 & 65 \\
\hline Watering eyes & 41 & 33 & 66 & 25 & 22 & 42 & 47 & 58 \\
\hline Vomiting & 47 & 39 & 37 & 29 & 59 & 15 & 33 & 42 \\
\hline \multicolumn{9}{|l|}{ Other symptoms (\%): } \\
\hline Photophobia & 41 & 28 & 43 & 31 & 30 & 24 & 64 & $35 \ddagger$ \\
\hline "Hard to open eyes" & 24 & 6 & 9 & 17 & 9 & 4 & 20 & 10 \\
\hline Diarrhoea & 12 & 6 & 9 & 8 & 4 & 2 & 0 & 0 \\
\hline Short of breath & 6 & 6 & 9 & 23 & 12 & 12 & 35 & 25 \\
\hline Chest pain & 6 & 11 & 14 & 17 & 10 & 10 & 16 & 25 \\
\hline Nausea & 0 & 39 & 1 & 12 & 4 & 22 & 15 & 2 \\
\hline Unconsciousness & 0 & 0 & 4 & 0 & 3 & 2 & 4 & 0 \\
\hline Dizziness & 6 & 0 & 1 & 2 & 4 & 2 & 0 & 0 \\
\hline Choking & 0 & 0 & 0 & 4 & 1 & 4 & 11 & 6 \\
\hline Headache & 0 & 6 & 0 & 2 & 3 & 0 & 0 & 0 \\
\hline Twitching & 0 & $\mathbf{0}$ & 0 & 0 & 4 & 0 & 2 & 0 \\
\hline Convulsions & 0 & 0 & 0 & 2 & 1 & 0 & 0 & 0 \\
\hline
\end{tabular}

* Number reported dead in each household visited divided by total examined plus number reported dead.

†Number of deaths not known.

†Includes both voluntered information and direct questioning.

$2 \cdot 3 ; \mathrm{OR}=3 \cdot 1)$ were more common in the non-exposed population. These differences were calculated over all age groups and disappeared with age stratification. There was an increase in fundal changes (dilated retinal vessels or haemorrhages) in the clusters where deaths had occurred (clusters 1-6: 80/272) by comparison with those where no deaths had occurred (clusters 7 and 8: 18/103). Data on four subjects were missing. These findings indicate a positive association, significant at the $2.5 \%$ level, between clusters where deaths occurred and fundal changes on ophthalmoscopy $\left(\chi^{2}=5 \cdot 5 ;\right.$ OR $\left.=1.97 ; 97.5 \% \mathrm{CI}=1.03-3 \cdot 76\right)$.

Unfortunately, the respiratory examination was omitted or no record was made for $55 \%$ of exposed survivors. Among the $45 \%$ who were examined, 25 $(15 \%)$ were judged to be in "respiratory distress" on the grounds of a conspicuously increased respiratory rate, an audible wheeze at rest, or use of the respiratory accessory muscles. Overall, there was no hint of an association between fundal changes and obvious respiratory distress $\left(\chi^{2}=0.04\right)$, which probably indicates lack of representativeness of the available $45 \%$ of the respiratory examination data. Considering only those people on whom a respiratory examination was recorded, 16 were considered to be in obvious respiratory distress out of the 131 who were normal on fundoscopy and nine were deemed to be in respiratory distress out of the $\mathbf{4 0}$ with abnormal fundi. Fundal changes were thus twice as common among people deemed to be in respiratory distress. This finding, however, was significant only at the $12 \%$ level $\left(\chi^{2}=\right.$ 2.6; OR $=2.09$, for $1 \mathrm{DF})$. Respiratory distress was observed nearly six times more often in clusters 1-6 where deaths had occurred (22/105) than in the other

Table 4 Signs on physical examination

\begin{tabular}{|c|c|c|c|c|c|c|c|c|c|c|}
\hline $\begin{array}{l}\text { Cluster } \\
\text { No }\end{array}$ & $\begin{array}{c}1 \\
70\end{array}$ & $\begin{array}{r}2 \\
17\end{array}$ & $\begin{array}{r}3 \\
69\end{array}$ & $\begin{array}{r}4 \\
50\end{array}$ & $\begin{array}{r}5 \\
52\end{array}$ & $\begin{array}{r}6 \\
18\end{array}$ & $\begin{array}{r}7 \\
55\end{array}$ & $\begin{array}{r}8 \\
48\end{array}$ & $\begin{array}{r}9 \\
65\end{array}$ & $\begin{array}{l}10 \\
54\end{array}$ \\
\hline Normal & 30 & 6 & 48 & 19 & 37 & 2 & 21 & 33 & 44 & 30 \\
\hline $\begin{array}{l}\text { Red eye } \\
\text { SIPE* } \\
\text { Bitot spot } \\
\text { Pterygium } \\
\text { Corneal opacity } \\
\text { Cataract } \\
\text { Discharge } \\
\text { Trachoma }\end{array}$ & $\begin{array}{r}23 \\
9 \\
5 \\
4 \\
6 \\
1 \\
1 \\
-\end{array}$ & $\begin{array}{l}4 \\
1 \\
1 \\
5 \\
-\end{array}$ & $\begin{array}{l}6 \\
9 \\
3 \\
4 \\
5 \\
- \\
-\end{array}$ & $\begin{array}{r}6 \\
18 \\
2 \\
3 \\
3 \\
3 \\
2\end{array}$ & $\begin{array}{l}5 \\
6 \\
1 \\
4 \\
2 \\
1 \\
1\end{array}$ & $\begin{array}{l}6 \\
9 \\
3 \\
1 \\
1 \\
1\end{array}$ & $\begin{array}{r}18 \\
2 \\
1 \\
14 \\
7 \\
- \\
- \\
-\end{array}$ & $\begin{array}{l}\frac{-}{1} \\
\frac{3}{1} \\
\frac{1}{6}\end{array}$ & $\begin{array}{l}6 \\
4 \\
9 \\
5 \\
3\end{array}$ & $\begin{array}{r}9 \\
2 \\
17 \\
4 \\
3 \\
3\end{array}$ \\
\hline $\begin{array}{l}\text { Respiratory distress } \\
\text { Examined }\end{array}$ & $\begin{array}{r}8 \\
18\end{array}$ & $\begin{array}{r}l \\
16\end{array}$ & $\begin{array}{r}4 \\
10\end{array}$ & $\begin{array}{r}3 \\
10\end{array}$ & $\begin{array}{r}6 \\
18\end{array}$ & $\begin{array}{r}0 \\
11\end{array}$ & $\begin{array}{r}1 \\
43\end{array}$ & $\begin{array}{r}2 \\
23\end{array}$ & & \\
\hline
\end{tabular}

*SIPE, superficial interpalpebral erosion.

Note: The number of signs in each cluster may exceed the total number of people in the cluster. Some individuals exhibited more than one sign. People, not eyes, were counted-for example, four red eyes equal four people with one or two red eyes each. 
Table 5 Relation between early eye symptoms and clinical eye damage (Cluster number, in descending order)

\begin{tabular}{lllllllll}
\hline & \multicolumn{1}{l}{ Rank } \\
\cline { 2 - 8 } & 1 & 2 & 3 & 4 & 5 & 6 & 7 & 8 \\
\hline Frequency of: & 7 & 8 & 1 & 5 & 2 & 3 & 6 & 4 \\
$\quad$ Burning & 7 & 1 & 2 & 8 & 5 & 3 & 6 & 4 \\
Photophobia & 2 & 7 & 5 & 8 & 1 & 3 & 6 & 4 \\
Difficulty in opening eyes & 6 & 4 & 3 & 1 & 5 & 2 & 7 & 8 \\
SIPE & &
\end{tabular}

SIPE, superficial interpalpebral erosion.

two exposure clusters $(3 / 66)$, a finding that was significant at the $0.5 \%$ level $\left(\chi^{2}=9.3\right.$; OR $=5.8$; $99 \cdot 5 \%$ CI $=1 \cdot 15-29 \cdot 6)$. No abnormal afferent pupillary reflex was detected in any of the clusters.

\section{Discussion}

The urgency of implementing this study so soon after the disaster, in substantially less than ideal working conditions, imposed several obvious defects in the data set. It is difficult to extrapolate from the number of deaths reported in this community based survey to the likely number of overall deaths that resulted from the disaster. Although the household survey in each cluster was started from an arbitrarily selected house, after which it proceeded from door to door, there may have been a tendency for some of the teams to move towards the worst affected areas in each community. Overall, however, the patterns of death were consistent with the reported wind directions: first northerly then moving westerly. The highest mortality was observed close to the plant and on the south east side, presumably reflecting the change in wind direction. Allowing for the varying levels of exposure across the reported 100000 people in communities with quite different density of housing, the official estimate of 2000 to 2500 deaths seems consistent with these data.

Perhaps the most striking impression from the affected communities in the days immediately after the episode was the large number of people with conspicuous difficulty in breathing. Some of these had been too disabled even to attend the local hospitals for treatment. During the two month follow up it was noted, again informally, that many of the children seen during the first visit still had conspicuous breathing difficulties. Such necropsy reports as are available indicate that some deaths were related to acute pulmonary oedema. Failure to record some basic data on respiratory status for the whole study population lost the unique opportunity offered by this survey, carried out so soon after the event. The afferent pupillary reflex indicates damage to the optic nerve and impairment of this reflex could be taken as evidence of neurotoxicity. The fact that this reflex was normal in all groups cannot be taken as evidence that neurotoxicity did not occur. The test may provide a useful baseline, however, for study over a longer period.

Our analysis of symptoms related only to the clusters where the deaths occurred, not to the individuals, so their interpretation in this context is limited. During the longer term follow up of survivors now in progress the predictive value of these symptoms in relation to prognosis could possibly be tested. The reported mortality in this study involved relatively small numbers in each cluster. None the less, the difference between clusters may provide a basis for further inquiries into the distribution and frequency of immediate and delayed deaths and the prognosis of symptoms among survivors.

The association of high death rates with reported cough and diarrhoea among the first four symptoms is difficult to reconcile with the apparently inverse relation between clusters where death was common and those where shortness of breath was a frequent symptom among survivors. A similarly curious finding was the inverse relation between reported burning of the eyes as an early symptom of exposure and the clinical finding of SIPE. Clusters 4 and 6, where SIPE was most commonly observed and burning of the eyes least frequently reported, were investigated by two different teams. This implies that the apparently inverse relation is not the result of an observer induced bias.

In some clusters the death rates may have been high enough for most people who had respiratory symptoms to have died, leaving alive only those who were relatively free of symptoms. If this was so the apparently direct relation with another respiratory symptom (cough) would be difficult to explain. It could be argued that the excess SIPE observed in survivors from the clusters where deaths had occurred could result from their running away from the exposure, as was done by many thousands of people. This might increase the exposure of their eyes and, theoretically, their chances of survival. In reality, most people who ran away went southward from the plant. They thus moved with the cloud, not away from it, which would probably reduce rather than increase their chances of survival. There was also no evidence of a difference between clusters where death rates were high and other exposed clusters in respect of pattern of exodus immediately after the release of gas.

The second and more plausible explanation is that the relation between dose and overall response is more complex than the usual log-linear relation. There appear to be at least two relatively distinct symptom/ pathology complexes which result from the exposure (see tables 3 and 4). This could be because different pathological reactions occur at different doses.

It is theoretically possible that the gas cloud may have had different constituent components after a certain time or in certain regions. We have shown that 
methyl isocyanate can react with methylamine, the product of its initial reaction with water to produce dimethyl urea. ${ }^{2}$ Various other oligomeric or polymeric adducts have been identified in the residues in the storage tank (S Varadarajan, 1985). Any of these could have had effects that are distinct from those of MIC. There could also be reactions between endogenous substances and MIC or its breakdown products. Thus people living in different areas could have symptom/pathology patterns relating to exposure to different chemical species. A counterindication to this suggestion is our observation that although liquid phase reactions between MIC and water are rapid, the vapour reactions are (as would be expected) much slower and consistent with the persistence of a concentrated cloud of MIC in its original form for some hours. ${ }^{2}$

On the other hand, laboratory toxicological experiments have shown distinct symptom/pathology groups at different dose levels for animals exposed to pure and continuously generated atmospheres of MIC. ${ }^{5}$ Broadly speaking, these may be classified into neuropharmacological (anaesthesia and increased sensitivity to subsequent anaesthesia), eye irritation and damage (including a form of SIPE similar to that seen in Bhopal survivors), and lung damage (acute bronchoconstriction and tachypnoea, pulmonary oedema, and in the longer term, fibrotic and inflammatory reactions). All these effect groups occurred at characteristic and different exposure levels: in particular, sensory and neurological responses were noticed at relatively low doses, the eye damage was apparent at intermediate exposures, and the more obvious and life threatening manifestations of pulmonary damage appeared at higher dose levels. If these differences are considered capable of extrapolation to the responses seen in the Bhopal survivors the differing distribution of symptoms between exposed groups (and by implication, severity of exposure) may be interpreted as the complex response to varying degrees of exposure in a gas cloud composed mainly or entirely of MIC.

The surface of the eye is moist under ordinary circumstances, so damage to this by a substance that is reactive with water ${ }^{2}$ will be associated with damage to other moist surfaces, including those of the lungs. The survivors in clusters where deaths had occurred were indeed at 12 times the risk of SIPE and six times the risk of respiratory distress compared with other exposed people. This leads us to the tentative conclusion that the deaths resulting from the exposure, reportedly pulmonary oedema, are associated geographically with cough and diarrhoea as early symptoms, and with SIPE and respiratory distress as clinical findings among the survivors.

The picture emerging from symptom patterns may be confounded by the fact that only the first four symptoms were requested. Later symptoms, however important their relation to pathology, were not recorded. It should also be reiterated that these initial symptoms were not directly associated with death of the individual with the symptom but only with the areas where death rates were higher.

Despite these caveats, there appear to be several geographical contrasts in the survivor population of Bhopal. The most obvious contrast is between the exposure and non-exposure clusters. Secondly, there appears to be a contrast in findings between clusters where deaths occurred (1-6) and the remaining two clusters. Thirdly, table 5 indicates that in certain clusters (notably 4 and 6) relatively few survivors reported burning eyes and shortness of breath, whereas cough, diarrhoea, respiratory distress, and SIPE were more common. In clusters $3,5,7$, and 8 deaths were more uncommon as were clinical findings of SIPE but a different pattern of symptoms was evident. Burning of the eyes, shortness of breath, photophobia, choking, twitching, and convulsions were all more frequent.

The long term follow up of survivors should allow us to test the predictive value of these signs and symptoms associated geographically with high death rates, information which may be of substantial value in preparing for and coping with the longer term health consequences of the disaster. The groups of symptoms with different patterns of death/survival in the various clusters implies that the gas has different effects on people at different concentrations (or at different distances from the factory). This appears to be a feature of the concentration dependent interaction of the gas and its reaction products with human tissue.

This work was partly funded by the Royal Commonwealth Society for the Blind. We acknowledge gratefully the help of Dr Kalyani Madan in execution of the field work. We are humbly indebted to the survivors of the disaster for their participation.

\section{References}

1 Andersson N, Kerr Muir M, Mehra V. Bhopal eye. Lancet 1984;ii:1481.

2 Andersson N, Kerr Muir M, Salmon AG, et al. Bhopal disaster: eye follow-up and analytical chemistry. Lancet 1985;i:761-2.

3 Mantel N, Haenszel W. Statistical aspects of the analysis of data from retrospective studies of disease. $J$ Natl Cancer Inst 1959;22:719-48.

4 Mantel N. Chi-square tests with one degree of freedom: extensions of the Mantel-Haenszel procedure. Journal of the American Statistical Association 1963;58:690-700.

5 Salmon AG, Kerr Muir M, Andersson N. Acute toxicity of methyl isocyanate. A preliminary study of the dose response for eye and other effects. Brit J Ind Med 1985;42:795-8. 